OPEN ACCESS

Edited by:

Taher Darreh-Shori, Karolinska Institutet (KI), Sweden

Reviewed by:

Frank Verbraak,

VU University Amsterdam,

Netherlands

Muhammet Erol,

Antalya IVF, Turkey

Jurre Den Haan,

VU University Amsterdam,

Netherlands

${ }^{*}$ Correspondence:

Zhaozeng Lu

Iuzc@shmu.edu.cn

Yiqin Xiao

xiaoyiqin1028@163.com

tThese authors have contributed equally to this work

Received: 14 June 2020 Accepted: 24 August 2020 Published: 15 January 2021

Citation:

Wang X, Zhao Q, Tao R, Lu H, Xiao $Z$, Zheng $L$, Ding $D$, Ding $S$,

Ma Y, Lu Z and Xiao Y (2021) Decreased Retinal Vascular Density in Alzheimer's Disease (AD) and Mild

Cognitive Impairment (MCl): An

Optical Coherence Tomography

Angiography (OCTA) Study.

Front. Aging Neurosci. 12:572484.

doi: 10.3389/fnagi.2020.572484

\section{Decreased Retinal Vascular Density in Alzheimer's Disease (AD) and Mild Cognitive Impairment (MCI): An Optical Coherence Tomography Angiography (OCTA) Study}

\author{
Xi Wang ${ }^{1 \dagger}$, Qianhua Zhao ${ }^{2,3+}$, Rui Tao', Huimeng Lu',3, Zhenxu Xiao ${ }^{2,3}$, Li Zheng ${ }^{2,3}$, \\ Ding Ding ${ }^{2,3}$, Saineng Ding ${ }^{2,3}$, Yichen Ma ${ }^{1}$, Zhaozeng $L u^{1 *}$ and Yiqin Xiao ${ }^{1 *}$
}

\footnotetext{
${ }^{1}$ Department of Ophthalmology, Huashan Hospital, Fudan University, Shanghai, China, ${ }^{2}$ Department of Neurology, Huashan Hospital, Fudan University, Shanghai, China, ${ }^{3}$ National Clinical Research Center for Aging and Medicine, Huashan Hospital, Fudan University, Shanghai, China
}

Background: To explore the retinal vascular density changes in Alzheimer's disease $(A D)$ and mild cognitive impairment $(\mathrm{MCl})$ patients using optical coherence tomography angiography (OCTA).

Methods: We recruit $62 \mathrm{AD}$ patients, $47 \mathrm{MCl}$ patients, and 49 cognitively healthy controls $(\mathrm{HC})$ in this study. All participants in the study received a comprehensive ophthalmological and neurological evaluation, including global cognitive screening, as well as the Mini-Mental State Examination (MMSE), and completed the following eye examinations: visual acuity (VA), intraocular pressure (IOP), examination with slitlamp, fundus photography (Version 1.5.0.0, NIDEK CO, LTD) and Optical coherence tomography imaging (software ReVue version 2017.1.0.155, Optovue Inc., Fremont, CA, United States). The visual rating scales for atrophy and white matter lesion in MRI was evaluated for all the patients with $\mathrm{AD}$ and $\mathrm{MCl}$.

Results: In the AD patient group, the superficial vascular density in the superior, inferior and whole retina was $44.64 \pm 3.34,44.65 \pm 3.55$, and $44.66 \pm 3.36$, respectively. These values were $44.24 \pm 3.15,43.72 \pm 3.16$, and $44 \pm 3.07$, respectively, in the $\mathrm{MCl}$ patient group. After multivariate analysis of the generalized linear model, adjustments for the confounding factors of sex, age, hypertension, diabetes and the quality index of OCTA image, the superficial vascular density in the $\mathrm{AD}$ and $\mathrm{MCl}$ patient groups was significantly lower than that in the $\mathrm{HC}$ group $(P<0.05): 46.94 \pm 2.04,46.67 \pm 2.26$, and $46.82 \pm 2.08$, respectively. No difference in the area of the FAZ among the three groups was observed (AD group: $0.34 \pm 0.11 \mathrm{~mm}^{2}$; $\mathrm{MCl}$ group: $0.36 \pm 0.12 \mathrm{~mm}^{2}$; control group: $\left.0.33 \pm 0.12 \mathrm{~mm}^{2}, p>0.05\right)$. The ganglion cell complex (GCC) thickness, inner parafovea thickness, and peripapillary retinal nerve fiber layer ( $p-R N F L)$ thickness were associated with the superficial vascular density. We found no significant correlation 
between the global cognition (MMSE scores) or between the Fazekas score and retinal OCT angiogram flow density.

Conclusion: The superficial vascular density in the $\mathrm{AD}$ and $\mathrm{MCl}$ patient groups was significantly lower than that in the $\mathrm{HC}$ group. Our findings suggest the retinal microvascular dysfunction occurred in $\mathrm{MCl}$ and $\mathrm{AD}$.

Keywords: Alzheimer's disease, mild cognitive impairment, optical coherence tomography angiography, retinal thickness, retinal blood flow

\section{INTRODUCTION}

Globally, approximately 47 million people suffer from dementia, and the number is expected to triple in 2050 (Livingston et al., 2017). China has the largest population of dementia patients in the world, imposing a heavy burden on the public and health-care system (Jia et al., 2020). Alzheimer's disease (AD), as the most common type of dementia, is characterized by its continuous, irreversible pathological process, which initiates decades before overt cognitive impairment (Sperling et al., 2011). Mild cognitive impairment (MCI) is the prodromal stage of $\mathrm{AD}$ (Morris et al., 2001; Langa and Levine, 2014). Individuals with MCI are at high risk of developing dementia. Although cerebrospinal fluid (CSF) analyses (amyloid and tau proteins) and positron emission tomography (PET) scans may help in the early identification of MCI (Albert et al., 2011; Ottoy et al., 2019), these methods have not been widely used in clinical practice due to their invasiveness and high cost (Handels et al., 2017). Economic, non-invasive, precise early markers are still needed.

Alzheimer's disease is also linked to many vascular risk factors, such as diabetes, stroke, atherosclerosis, and hypertension (Vos et al., 2015). A cross-sectional study involving 1,143 subjects showed that cerebral atherosclerosis and arteriosclerosis were associated with AD and dementia (Arvanitakis et al., 2016). Recent studies have found an association of cerebral blood flow (CBF) with the pathogenesis of $\mathrm{AD}$, suggesting that it may serve as a biomarker for preclinical AD (Hays et al., 2016). In patients with $\mathrm{MCI}$ and $\mathrm{AD}$, cerebral hypoperfusion has been reported to increase cerebral vascular tortuosity and decrease vascular density (Yamashita et al., 2014).

Although there is evidence that vascular injury plays a role in the pathogenesis of $\mathrm{AD}$ and $\mathrm{MCI}$, it is unclear whether vascular changes cause neurological death directly or whether they are secondary effects of reduced neuronal metabolism. However, the cerebral blood vessels are too small to be directly observed and assessed in vivo (Smith and Beaudin, 2018). Because the retinal and cerebral vasculatures are similar in embryonic development and physiological and anatomical features, researchers have focused on investigating intraocular vascular structures to understand cerebral vasculature (Berisha et al., 2007). Retinal Laser Doppler flowmetry has been used to detect venous stenosis and decreased blood flow in patients with AD and MCI (Feke et al., 2015).

Optical coherence tomography angiography (OCTA) is a new angiographic technology based on OCT technology that can visualize and quantify retinal and choroidal vessels without intravenous injection of contrast agent. It can provide highresolution images of retinal and choroidal microvasculature to visualize the changes in retinal blood vessels. OCTA has more advantages than fundus fluorescein angiography (FFA) and Retinal Laser Doppler flowmetry. It is non-invasive, safer, and faster. It can better display the superficial and deep capillary layers of the retina and can quantitatively measure the non-perfused area of the macula.

Optical coherence tomography angiography capillary perfusion density map and mean perfusion density provide a simple method for grading the gradual changes in blood vessels. During the last 2 years, OCTA has been used in many ocular and systemic diseases. Bulut et al. (2018) were the first to use OCTA to assess retinal vascular changes in AD. They found that vessel density was significantly reduced in all regions of the $\mathrm{AD}$ group $(P<0.05)$, and this reduction was correlated with Mini-Mental State Examination (MMSE) scores (Bulut et al., 2018). Many studies have found that vessel density decreases in the deep vascular plexus (DVP) and superficial vascular plexus (SVP) in AD patients (Jiang et al., 2018; Zabel et al., 2019b). In particular, the reduced blood flow density of the retina was related to the cerebrovascular disease of $\mathrm{AD}$ but not to the pathology of $\mathrm{AD}$ (Lahme et al., 2018). However, there are also different findings. van de Kreeke et al. (2020) found that $A \beta+$ individuals had significantly higher vessel density than $A \beta-$ individuals in all regions. At present, only a few studies pay attention to the changes in retinal blood flow in MCI. Jiang et al. (2018) found that the density of retinal microvessels of the DVP in the superior nasal quadrant was significantly lower in patients with MCI than in the control group.

This study aimed to evaluate retinal macular area and optic nerve head $(\mathrm{ONH})$ perfusion in patients with MCI and early $\mathrm{AD}$ using OCTA. In addition, we quantified the association of OCTA findings with retinal thickness. A direct comparison of OCTA analysis in patients with $\mathrm{AD}$ and MCI could make a significant contribution to understanding the pathophysiology of these neurodegenerative diseases and may help to elucidate the underlying cause of the thinning of the peripapillary retinal nerve fiber layer ( $p-R N F L)$. We hypothesized that retinal vascular pathology contributes to downstream neurodegeneration. We wanted to explore whether retinal blood flow changes in $\mathrm{MCI}$ and early $\mathrm{AD}$ patients and, if so, whether this change correlates with changes in retinal nerve thickness and with cognitive scores and cerebrovascular lesions. 


\section{MATERIALS AND METHODS}

\section{Participants}

A total of $47 \mathrm{MCI}$ patients, 49 age-matched healthy controls (HC), and $62 \mathrm{AD}$ patients participated in this study. The patients were enrolled from the memory clinic in Huashan Hospital from December 2017 to March 2018. HC subjects were recruited from the Shanghai Aging Study, a community-based aging cohort. $\mathrm{AD}$ and MCI were diagnosed based on the clinical evaluation, neuropsychological assessment, neuroimaging (such as cranial MR or CT), and laboratory tests according to the 2011 guidelines of the National Institute of Aging-Alzheimer's Association workgroups (NIA/AA). The HC subjects had no evidence of neurological disorders and were determined to be cognitively healthy according to neuropsychological assessment. The interval between the ophthalmic examination and clinical evaluation was 1-90 days. The study was approved by the Huashan
Hospital Institutional Review Board (HIRB). All participants were informed of the study protocol in detail and signed the informed consent form. All participants were treated under the tenets of the Declaration of Helsinki.

All participants in the study underwent global cognitive screening, as well as the MMSE, and completed the following eye examinations: visual acuity (VA), intraocular pressure (IOP), examination with slit-lamp, fundus photography (Version 1.5.0.0, Nidek Co, Ltd) and OCTA. Patients with MCI or AD had to undergo a comprehensive battery of tests that covered four main domains: (1) memory, (2) executive function/attention, (3) language, and (4) visuospatial skills. Normative data and detailed descriptions of these tests are reported elsewhere (Karakosta et al., 2012; Xu et al., 2018). MCI and AD were diagnosed based on the 2011 NIA/AA guidelines. The HC subjects had no evidence of a neurological disorder or cognitive complaints.
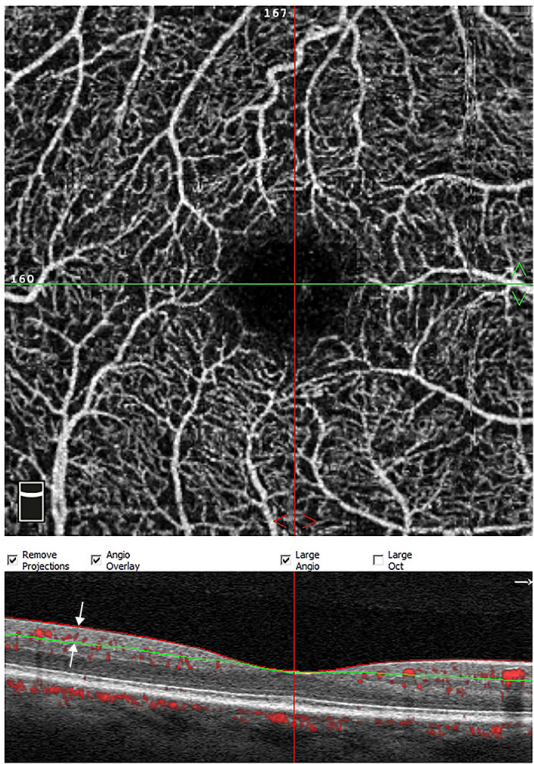

A Superficial

\begin{tabular}{|l|l|l|}
\hline $\begin{array}{l}\text { ETDRS Grid } \\
\text { Parameters }\end{array}$ & Hemisphere & Full Ring \\
\hline & \\
\hline
\end{tabular}

(D) Zoning Diagram

B Deep

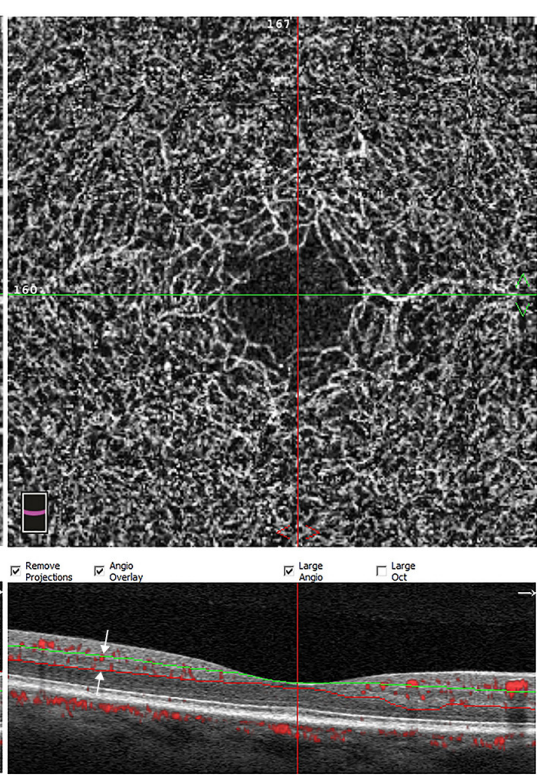

Vessel Density \& OCT Thickness ILM-RPE
\begin{tabular}{|cll|}
\hline Density $(\%)$ & Section & Thickness $(\mu \mathrm{m})$ \\
\hline 48.7 & Whole Image & 316 \\
48.7 & Superior-Hemi & 315 \\
48.8 & Inferior-Hemi & 317 \\
17.0 & Fovea & 258 \\
52.0 & ParaFovea & 326 \\
\hline
\end{tabular}

(E) Data Diagram

FIGURE 1 | (A) is the superficial en face image, which was segmented with an inner boundary at the internal limiting membrane (red line) and an outer boundary set at $10 \mathrm{~mm}$ above the inner plexiform layer (green line). (B) is the deep en face image, which segmented with an inner boundary at $10 \mathrm{~mm}$ above the inner plexiform layer (green line) and an outer boundary at $10 \mathrm{~mm}$ beneath the outer plexiform layer (red line). (C) is the radial peripapillary capillaries (RPCs), which was defined as extending from the internal limiting membrane to the posterior boundary of the RNFL. (D) is Zoning diagram two different modes of data analysis: hemisphere and full ring. (E) is data diagram. All scans were reviewed independently by two investigators to ensure correct segmentation and sufficient image quality. 
For all groups, the exclusion criteria were as follows: (1) personal or family history of psychiatric disorders, Lewy body dementia, vascular dementia, frontal temporal dementia, multiple sclerosis, or Parkinson's disease,; (2) a history of glaucoma, retinal detachment, optic neuropathy, other optic nerve diseases and retinal vascular disorders such as agerelated macular degeneration, ocular trauma, a cataract that disturbs visual and OCT examination, or other ocular disorders; (3) a history of carbon monoxide poisoning, alcohol abuse, hypothyroidism, or other serious chronic medical conditions; and (4) severe cognitive impairment rendering the individual unable to cooperate in the eye examination.

\section{Optical Coherence Tomography Imaging}

Optical coherence tomography angiography imaging of all subjects was performed with the Optovue Angiovue System (software ReVue version 2017.1.0.155, Optovue Inc., Fremont, CA, United States). The device obtains volumetric scans of $304 \times 304 \mathrm{~A}$-scans at 70,000 A-scans per second in approximately $3.0 \mathrm{~s}$. Two B-scans were captured at each fixed position before proceeding to the next sampling location, and two orthogonal OCTA volume scans were used to minimize motion artifacts and fixation changes. Macula imaging was performed using a $3 \times 3 \mathrm{~mm}^{2}$ scan and images of the $\mathrm{ONH}$, and the peripapillary area was obtained with a $4.5 \times 4.5 \mathrm{~mm}^{2}$ scan. The software automatically segmented and calculated these full-thickness retinal scans into the superficial and deep inner retinal vascular plexuses, outer retina, and choriocapillaris. Each $3 \times 3-\mathrm{mm}$ parafoveal scan area was automatically segmented as follows: The superficial en face image was segmented with an inner boundary at the internal limiting membrane and an outer boundary set at $10 \mathrm{~mm}$ above the inner plexiform layer, whereas the deep en face image was segmented with an inner boundary at $10 \mathrm{~mm}$ above the inner plexiform layer and an outer boundary at $10 \mathrm{~mm}$ beneath the outer plexiform layer. All scans were reviewed independently by two investigators (XW and RT) to ensure correct segmentation and sufficient imaging quality (Figures 1A,B). The foveal avascular zone (FAZ) was also automatically identified.

Macular vessel density was analyzed throughout the area of $3 \times 3 \mathrm{~mm}^{2}$. Two different modes of data analysis were used: (1) the software automatically divided the macular retina into superior and inferior regions and (2) two circular areas from the center to the outside were calculated, the fovea $(\varnothing=1 \mathrm{~mm})$ and the parafovea $(\varnothing=3 \mathrm{~mm}$ ) (Figures 1D,E). The density of the radial peripapillary capillaries (RPCs) was defined as extending from the internal limiting membrane to the posterior boundary of the RNFL (Figure 1C). We used the Optovue AngioVue System to investigate retinal thickness by measuring the following indexes: p-RNFL thickness and ganglion cell complex (GCC) thickness.

All the measurements were completed by one ophthalmologist. Images were independently reviewed by two ophthalmologists, and only those with good quality were included (image quality $>6$ ). Measurements with motion artifacts present on the en face images were also rejected. For each participant, both eyes were examined. However, to ensure the independence of variables, calculated means of eye results were included in the statistical analysis. The right or left eye was chosen if the other eye was ineligible. The ophthalmologists were blinded to the diagnosis in both the image review phase and the data analysis phase.

\section{Neuroimaging Data Acquisition and Imaging Analysis}

To ensure the uniformity of data collection, we performed brain MR imaging through standard procedures. A brain scan was conducted in a Siemens $3.0 \mathrm{~T}$ MAGNETOM Verio with an MPRAGE T1WI sequence. The voxel size was $1 \times 1 \times 1 \mathrm{~mm}$. The FreeSurfer ${ }^{1}$ Analysis software package was used to analyze and visualize structural neuroimaging data obtained from cross-sectional or longitudinal views. The procedures used for MRI structural analysis were as follows: (1) OsiriX ${ }^{2}$ software was used to view the obtained brain MRI structure and exclude data produced by apparent lesions and imaging artifacts; (2) FreeSurfer software was used to analyze the brain MRI structure screened by reconall instructions; (3) tKmedit and tKsurfer were used to check the partition of white-gray matter after checking recon-all.log to exclude any errors; and (4) Asegstats2table and aparcstats2table were used to extract data for the relevant structure.

The examination timepoints of OCTA and MRI scans were within a six-month period. The Fazekas scale was independently used by two experienced observers who were blinded to clinical

\footnotetext{
${ }^{1}$ http://surfer.nmr.mgh.harvard.edu

${ }^{2} \mathrm{http} / / /$ www.osirix-viewer.com
}

TABLE 1 | Demographic and clinical characteristics of the study subjects.

\begin{tabular}{|c|c|c|c|c|}
\hline & $\begin{array}{l}\text { Patients } \\
\text { with } A D\end{array}$ & $\begin{array}{l}\text { Patients } \\
\text { with } \mathrm{MCl}\end{array}$ & $\begin{array}{l}\text { Control } \\
\text { subjects }\end{array}$ & $p$-value \\
\hline & $(n=62)$ & $(n=47)$ & $(n=49)$ & \\
\hline Age & $71.81 \pm 7.98$ & $72.73 \pm 7.75$ & $69.50 \pm 5.94$ & 0.079 \\
\hline Medium (range) & $73(57,89)$ & $74(53,87)$ & $71(57,81)$ & \\
\hline $\operatorname{Sex}(M / F)$ & $27 / 35$ & $18 / 29$ & $17 / 32$ & 0.634 \\
\hline VA & $0.68 \pm 0.25$ & $0.75 \pm 0.22$ & $0.70 \pm 0.14$ & 0.275 \\
\hline IOP (mmHg) & $14.24 \pm 2.55$ & $14.62 \pm 2.57$ & $14.26 \pm 2.20$ & 0.718 \\
\hline $\mathrm{HBP}$ & 22 & 19 & 18 & 0.865 \\
\hline DM & 8 & 8 & 8 & 0.812 \\
\hline MMSE score & $19.92 \pm 4.54$ & $28.04 \pm 1.90$ & $28.67 \pm 1.0$ & $<0.001^{\star *}$ \\
\hline Medium (range) & $20(7,24)$ & $28(23,30)$ & $29(27,30)$ & \\
\hline CDR global & $1.339 \pm 0.061$ & $0.532 \pm 0.018$ & $0.031 \pm 0.017$ & $<0.001^{\star *}$ \\
\hline MTA & $1.77 \pm 0.231$ & $1.34 \pm 0.143$ & $0.89 \pm 0.200$ & 0.071 \\
\hline Fazekas & $0.88 \pm 0.234$ & $0.71 \pm 0.137$ & $0.56 \pm 0.176$ & 0.598 \\
\hline GCA & $1.46 \pm 0.144$ & $1.14 \pm 0.147$ & $0.780 \pm 0.147$ & 0.108 \\
\hline PCA & $1.08 \pm 0.178$ & $0.76 \pm 0.137$ & $0.44 \pm 0.176$ & 0.257 \\
\hline
\end{tabular}

Data reported as mean and $S D$ (standard deviation) with $p$ values. AD, Alzheimer's disease; $M C l$, mild cognitive impairment; $M$, male; $F$, female; $V A$, visual acuity; IOP, intraocular pressure; HBP, high blood pressure; DM, diabetes mellitus; MMSE, Mini-Mental State Examination; CDR, clinical dementia rating, the global score of $C D R$ was used. The bold values indicate statistical significance. ${ }^{* *} p<0.01$. 
information. A $0-3$ rating scale was used to quantify the amount of white matter T2 hyperintense lesions, which are often attributed to chronic small vessel ischemia (Fazekas et al., 1987; Scheltens et al., 1998).

\section{Statistics}

IBM SPSS Statistics software (vision 24) was used in the data analysis. Continuous parameters are expressed as the mean and the standard deviation (SD), and categorical parameters are shown as number and frequencies (\%). Data were tested for normal distribution by histograms and Q-Q plots. Normally distributed data were analyzed with one-way ANOVA, non-normally distributed data were analyzed with a MannWhitney $U$ test, and binary variables were analyzed with Fisher's exact test. For multiple comparisons, Bonferroni correction was used if necessary. The post hoc test was adopted for the ANOVA, and the Dwass, Steel, Critchlow-Fligner multiple comparison procedure (DSCF) was used for the Kruskal-Wallis test. Linear regression was used to assess the association between OCTA and retinal thickness measures. The generalized linear model (GLM) was used to evaluate the association between the study groups and the OCTA measures to adjust for age, sex, hypertension, diabetes and the quality index of OCTA image. Two-sided $p$-values and $95 \%$ CIs were used in SPSS software. Significance was determined at $p<0.05$.

TABLE 2 | Comparison of vascular density in macular among the three groups.

\begin{tabular}{|c|c|c|c|c|c|}
\hline & \multirow{2}{*}{$\frac{\text { Patients with AD }}{\text { Mean } \pm \text { SD }}$} & \multirow{2}{*}{$\frac{\text { Patients with } \mathrm{MCl}}{\text { Mean } \pm \text { SD }}$} & \multirow{2}{*}{$\frac{\text { Healthy control subjects }}{\text { Mean } \pm \text { SD }}$} & \multicolumn{2}{|c|}{$p$-value } \\
\hline & & & & $A D$ vs $H C$ & $\mathrm{MCl}$ vs $\mathrm{HC}$ \\
\hline \multicolumn{6}{|c|}{ OCT-A deep (\%) } \\
\hline Whole enface & $49.42 \pm 3.4$ & $49.57 \pm 2.89$ & $50.89 \pm 2.86$ & $0.016^{\star}$ & $0.041^{*}$ \\
\hline Superior & $49.53 \pm 3.48$ & $49.69 \pm 2.84$ & $50.98 \pm 2.89$ & $0.018^{*}$ & $0.049^{\star}$ \\
\hline Inferior & $49.36 \pm 3.51$ & $49.46 \pm 3.05$ & $50.78 \pm 2.89$ & $0.023^{\star}$ & $0.046^{\star}$ \\
\hline Fovea & $28.53 \pm 6.8$ & $26.83 \pm 7.11$ & $28.94 \pm 6.7$ & 0.758 & 0.137 \\
\hline Parafovea & $52.02 \pm 3.65$ & $52.36 \pm 2.96$ & $53.4 \pm 2.77$ & $0.027^{\star}$ & 0.118 \\
\hline \multicolumn{6}{|c|}{ OCT-A superficial (\%) } \\
\hline Whole enface & $44.66 \pm 3.36$ & $44 \pm 3.07$ & $46.82 \pm 2.08$ & $<0.001^{\star *}$ & $<0.001^{\star *}$ \\
\hline Superior & $44.64 \pm 3.34$ & $44.24 \pm 3.15$ & $46.94 \pm 2.04$ & $<0.001^{\star \star}$ & $<0.001^{\star *}$ \\
\hline Inferior & $44.65 \pm 3.55$ & $43.72 \pm 3.16$ & $46.67 \pm 2.26$ & $0.001^{* *}$ & $<0.001^{\star *}$ \\
\hline Fovea & $15.89 \pm 5.34$ & $14.09 \pm 5.21$ & $16.18 \pm 5.27$ & 0.776 & 0.057 \\
\hline Parafovea & $47.7 \pm 3.76$ & $47.12 \pm 3.35$ & $49.86 \pm 2.26$ & $0.001^{* *}$ & $<0.001^{\star \star}$ \\
\hline $\mathrm{FAZ}\left(\mathrm{mm}^{2}\right)$ & $0.34 \pm 0.11$ & $0.36 \pm 0.12$ & $0.33 \pm 0.12$ & 0.937 & 0.271 \\
\hline
\end{tabular}

Data reported as mean and SD (standard deviation) with $p$ values. The bold values indicate statistical significance. ${ }^{*} p<0.05$ and ${ }^{* *} p<0.01$.

TABLE 3 | Multivariate analysis of retinal vascular density.

\begin{tabular}{|c|c|c|c|c|c|c|}
\hline & \multicolumn{3}{|c|}{$A D$ versus $H C$} & \multicolumn{3}{|c|}{$\mathrm{MCl}$ versus $\mathrm{HC}$} \\
\hline & $\beta$ & $95 \% \mathrm{Cl}$ & p-value & $\beta$ & $95 \% \mathrm{Cl}$ & p-value \\
\hline \multicolumn{7}{|l|}{ OCT-A deep } \\
\hline Whole enface & -0.2616 & $(-0.8053,0.2821)$ & 0.3456 & -0.7413 & $(-1.7874,0.3049)$ & 0.1649 \\
\hline Superior & -0.2539 & $(-0.8085,0.3007)$ & 0.3695 & -0.7088 & $(-1.7471,0.3294)$ & 0.1809 \\
\hline Inferior & -0.2365 & $(-0.7966,0.3237)$ & 0.4080 & -0.7479 & $(-1.8377,0.3418)$ & 0.1786 \\
\hline Fovea & -0.0276 & $(-1.3514,1.2962)$ & 0.9674 & -2.3619 & $(-5.1648,0.4410)$ & 0.0986 \\
\hline Parafovea & -0.2793 & $(-0.8427,0.2840)$ & 0.3311 & -0.5114 & $(-1.5594,0.5365)$ & 0.3388 \\
\hline \multicolumn{7}{|c|}{ OCT-A superficial } \\
\hline Whole enface & -0.7020 & $(-1.2254,-0.1787)$ & $0.0086^{\star \star}$ & -2.0889 & $(-2.9873,-1.1906)$ & $<0.0001^{\star *}$ \\
\hline Superior & -0.7614 & $(-1.2756,-0.2472)$ & $0.0037^{\star \star}$ & -1.9547 & $(-2.8576,-1.0518)$ & $<0.0001^{\star *}$ \\
\hline Inferior & -0.6394 & $(-1.2015,-0.0774)$ & $0.0258^{\star}$ & -2.2304 & $(-3.1875,-1.2734)$ & $<0.0001^{\star *}$ \\
\hline Fovea & 0.0082 & $(-1.0325,1.0488)$ & 0.9877 & -2.1499 & $(-4.2692,-0.0306)$ & $0.0468^{\star}$ \\
\hline Parafovea & -0.6451 & $(-1.2228,-0.0673)$ & $0.0286^{\star}$ & -1.9629 & $(-2.9529,-0.9730)$ & $0.0001^{\star \star}$ \\
\hline \multicolumn{7}{|l|}{ OCT-A disk } \\
\hline Whole enface & 0.1561 & $(-1.1079,1.4202)$ & 0.8087 & 0.6946 & $(-1.4836,2.8728)$ & 0.5320 \\
\hline Peripapillary & -0.3611 & $(-0.9918,0.2697)$ & 0.2619 & -0.5785 & $(-1.7105,0.5534)$ & 0.3165 \\
\hline
\end{tabular}



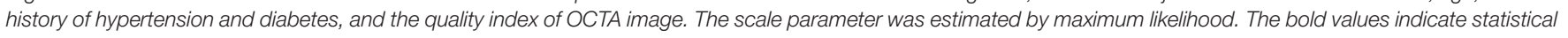
significance. ${ }^{*} p<0.05$ and ${ }^{* *} p<0.01$. 


\section{RESULTS}

\section{Characteristics}

A total of 191 subjects were referred for the study. After meeting the inclusion criteria, retinal blood flow and thickness measurements were successfully obtained in 165 subjects. Of the remaining subjects, seven of these individuals did not achieve OCTA image quality $>6$. Data from these subjects were excluded from the analysis. As a result, data from 158 subjects were used in the analysis.

The demographic and clinical characteristics of subjects in the $\mathrm{AD}, \mathrm{MCI}$, and HC groups are shown in Table 1. All groups were comparable in age, sex, VA, IOP, HBP, DM ratio, and visual rating scales (MTA, Fazekas, GCA and PCA) $(p>0.05)$. Compared with the MCI and $\mathrm{HC}$ groups, the AD cohort had a significantly worse MMSE score $(p<0.01)$.

\section{OCTA Findings}

The macular flow densities determined by retinal OCT angiogram are summarized in Table 2 . In the $\mathrm{AD}$ patient group, the superficial vascular density in the superior, inferior and whole retina was $44.64 \pm 3.34,44.65 \pm 3.55$, and $44.66 \pm 3.36$, respectively. These values were $44.24 \pm 3.15,43.72 \pm 3.16$, and $44 \pm 3.07$, respectively, in the MCI patient group. The superficial vascular density in the $\mathrm{AD}$ and MCI patient groups was significantly lower than that in the HC group $(P<0.05)$ : $46.94 \pm 2.04,46.67 \pm 2.26$, and $46.82 \pm 2.08$, respectively. In the $\mathrm{AD}$ patient group, the deep vascular density in the superior, inferior and whole retina was $49.53 \pm 3.48,49.36 \pm 3.51$, and $49.42 \pm 3.4$, respectively, while these values were $49.69 \pm 2.84$, $49.46 \pm 3.05$, and $49.57 \pm 2.89$, respectively in the MCI patient group. The deep vascular density in the AD and MCI patient groups was significantly lower than that in the $\mathrm{HC}$ group $(P<0.05): 50.98 \pm 2.89,50.78 \pm 2.89$, and 50.89 \pm 2.86 , respectively. No difference in the area of the FAZ among the three groups was observed (AD group: $0.34 \pm 0.11 \mathrm{~mm}^{2}$; MCI group: $0.36 \pm 0.12 \mathrm{~mm}^{2}$; control group: $0.33 \pm 0.12 \mathrm{~mm}^{2}, p>0.05$ ).

However, after multivariate analysis by a generalized linear model and after adjustments of the confounding factors of sex, age, hypertension, diabetes and the quality index of OCTA image, as shown in Table 3 and Figure 2, only the superficial vascular density in the $\mathrm{AD}$ and MCI patient groups was significantly lower than that in the HC group. The analysis of vessel density in the RPC layer on the whole en face image and the peripapillary area did not differ significantly among the three groups (AD group: $48.38 \pm 2.19$; MCI group: $48.65 \pm 1.92$; HC group: $48.33 \pm 6.99$, $p>0.05)$ in Tables 4,5 .

\section{Association Analysis}

The association between OCTA vascular density measures and retinal thickness is shown in Table 6. The variable was estimated by a linear regression model. We found a significantly positive association between vascular density measures and retinal thickness $(p<0.001)$. Moreover, we also observed a meaningful association between the inner parafoveal thickness and whole and parafoveal superficial vascular density $(p<0.001)$ (Table 6).
A

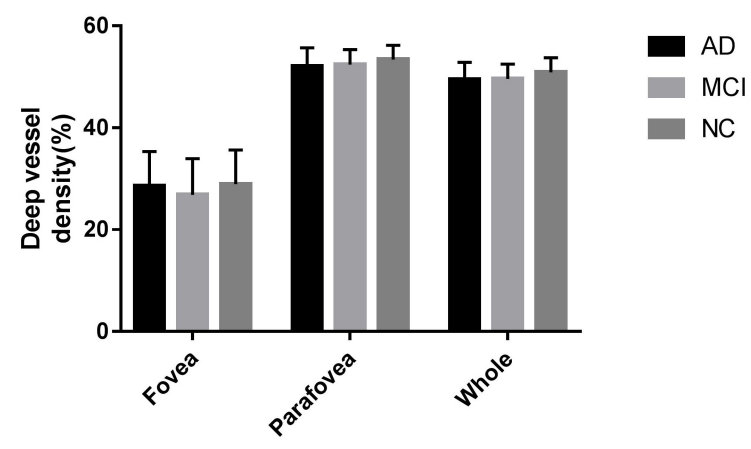

B

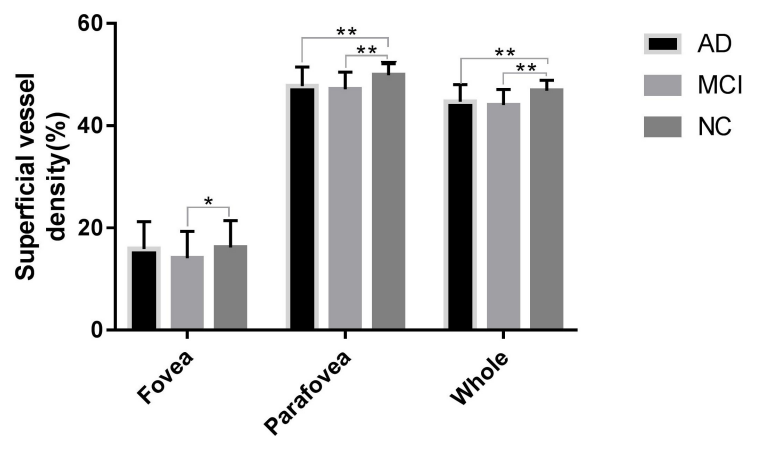

FIGURE 2 | Comparison of vascular density in the fovea, parafovea, and whole areas in the studied groups within the deep retinal vascular plexuses (A) and superficial retinal vascular plexuses (B). ${ }^{*} p<0.05$ and ${ }^{* *} p<0.01$.

Regarding the area of the $\mathrm{ONH}$, there was a positive correlation between $\mathrm{p}$-RNFL thickness and the parafovea superficial density $(p<0.001)$. The associations were adjusted for the confounders of sex, age, medical history of hypertension, diabetes and the quality index of OCTA image. The data are shown in Table 6.

The current study focused on patients with $\mathrm{AD}$ and MCI with few vascular burdens, among which the mean score of the Fazekas scale was $0.64 \pm 0.727$. We found no significant correlation between global cognition (MMSE scores) or between the Fazekas score and retinal OCT angiogram flow density (Table 7).

\section{DISCUSSION}

Cerebral small blood vessel disease is a risk factor for stroke and dementia (Rensma et al., 2018; van der Flier et al., 2018). More importantly, reduced CBF in MCI patients, compared with controls, has been reported to predict the progression to $\mathrm{AD}$ in longitudinal studies (Hirao et al., 2005; Hansson et al., 2009; Park et al., 2012). Moreover, the degree of the decrease in parietal rCBF, but not CSF biomarkers, was related to the more rapid development of AD (Hansson et al., 2009).

The retina is regarded as a part of the central nervous system. It is a direct extension of the brain during embryonic development. Our study shows that the superficial retinal blood 
TABLE 4 | Comparison of vascular density in $\mathrm{ONH}$ among the three groups.

\begin{tabular}{|c|c|c|c|c|c|}
\hline & \multirow{2}{*}{$\frac{\text { Patients with AD }}{\text { Mean } \pm \text { SD }}$} & \multirow{2}{*}{$\frac{\text { Patients with } \mathrm{MCl}}{\text { Mean } \pm \text { SD }}$} & \multirow{2}{*}{$\frac{\text { Healthy control subjects }}{\text { Mean } \pm \text { SD }}$} & \multicolumn{2}{|c|}{$p$-value } \\
\hline & & & & $A D$ vs $H C$ & $\mathrm{MCl}$ vs $\mathrm{HC}$ \\
\hline \multicolumn{6}{|c|}{ OCT-A disk (\%) } \\
\hline Whole enface & $48.38 \pm 2.19$ & $48.65 \pm 1.92$ & $48.33 \pm 6.99$ & 0.972 & 0.754 \\
\hline Inside disk & $51.48 \pm 3.5$ & $50.66 \pm 5.14$ & $49.72 \pm 4.6$ & 0.106 & 0.344 \\
\hline Peripapillary & $50.71 \pm 2.39$ & $50.91 \pm 2.33$ & $51.66 \pm 2.94$ & 0.123 & 0.187 \\
\hline
\end{tabular}

Data reported as mean and SD (standard deviation) with p values.

TABLE 5 | Multivariate analysis of $\mathrm{ONH}$ vascular density.

\begin{tabular}{|c|c|c|c|c|c|c|}
\hline & \multicolumn{3}{|c|}{$A D$ versus $H C$} & \multicolumn{3}{|c|}{$\mathrm{MCl}$ versus $\mathrm{HC}$} \\
\hline & $\beta$ & $95 \% \mathrm{Cl}$ & $p$-value & $\beta$ & $95 \% \mathrm{Cl}$ & $p$-value \\
\hline Inside disk & 1.8052 & $(-2.0813,0.414)$ & 0.0647 & 1.3238 & $(-0.7132,3.3607)$ & 0.2027 \\
\hline Whole enface & -0.1402 & $(-2.6865,2.4061)$ & 0.9141 & 0.5569 & $(-1.6346,2.7484)$ & 0.6184 \\
\hline Peripapillary & -0.8336 & $(-2.0813,0.414)$ & 0.1903 & -0.6387 & $(-1.7622,0.4848)$ & 0.2652 \\
\hline
\end{tabular}

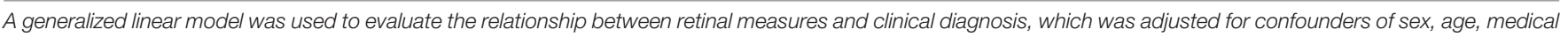
history of hypertension and diabetes and the quality index of OCTA image. The scale parameter was estimated by maximum likelihood.

TABLE 6 | Association between the retinal flow density and thickness.

\begin{tabular}{|c|c|c|c|c|c|c|c|c|}
\hline & \multicolumn{2}{|c|}{ Whole deep } & \multicolumn{2}{|c|}{ Parafovea deep } & \multicolumn{2}{|c|}{ Whole superficial } & \multicolumn{2}{|c|}{ Parafovea superficial } \\
\hline & $\beta$ & $p$-value & $\beta$ & $p$-value & $\beta$ & $p$-value & $\beta$ & $p$-value \\
\hline GCC thickness & -0.053 & 0.165 & -0.046 & 0.235 & 0.166 & $<0.001^{\star \star}$ & 0.164 & $<0.001^{\star *}$ \\
\hline Full fovea thickness & 0.015 & 0.267 & -0.002 & 0.862 & 0.016 & 0.216 & 0.007 & 0.607 \\
\hline Inner fovea thickness & 0.027 & 0.322 & -0.021 & 0.454 & 0.046 & 0.074 & 0.023 & 0.414 \\
\hline Outer fovea thickness & 0.021 & 0.354 & 0.007 & 0.763 & 0.010 & 0.621 & 0.003 & 0.881 \\
\hline Full parafovea thickness & 0.006 & 0.728 & 0.003 & 0.843 & 0.025 & 0.099 & 0.028 & 0.091 \\
\hline Inner parafovea thickness & 0.002 & 0.942 & -0.011 & 0.700 & 0.088 & $0.001^{* \star}$ & 0.093 & $0.001^{* *}$ \\
\hline Outer parafovea thickness & 0.018 & 0.520 & 0.024 & 0.407 & -0.010 & 0.705 & -0.007 & 0.801 \\
\hline p-RNFL thickness & -0.013 & 0.606 & -0.005 & 0.852 & 0.074 & $0.003^{\star \star}$ & 0.070 & $0.009^{\star *}$ \\
\hline
\end{tabular}





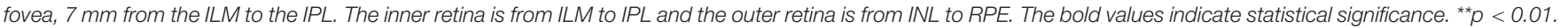

flow density of the macula under OCT angiography in the AD and MCI groups was significantly lower than that in the control group after adjustment for vascular factors such as diabetes and hypertension. Many other studies of the individual retinal vessel plexuses by OCTA showed that all regions of the SVP and DVP in $\mathrm{AD}$ patients had significantly lower retinal vascular density than healthy controls (Bulut et al., 2018; Jiang et al., 2018). However, Haan et al. (2019) found no differences in vessel density between amyloid-positive $\mathrm{AD}$ cases and control subjects by OCTA measurements. van de Kreeke et al. (2020) found that retinal vessel density was higher in individuals with preclinical AD. The inconsistent findings might be attributed to the variability of inclusion and exclusion criteria and different sample sizes, cognitive function tests, controls, and confounding factors.

Multiple studies have demonstrated that the retinal thickness of the GCC (Cheung et al., 2015; den Haan et al., 2018) and
p-RNFL (Kesler et al., 2011; Marziani et al., 2013; Pillai et al., 2016; Trebbastoni et al., 2016) is decreased in both AD and MCI patients. However, den Haan et al. (2018) found no retinal thinning in amyloid $+\mathrm{AD}$ patients. Further studies found that retinal thickness cannot discriminate between healthy individuals and preclinical AD individuals (den Haan et al., 2019a,b). In a recent study involving 930 participants (414 cognitively healthy people, 192 with probable amnestic MCI, and 324 probable $\mathrm{AD}$ patients), the results showed that there were no significant differences in retinal thickness in different layers (Sánchez et al., 2020). In one of our earlier articles, we found that the inner retinal layers of the macular area in MCI patients showed significant thinning compared with those in HCs (Tao et al., 2019). Furthermore, in this study, we found a significantly positive correlation between GCC thickness, p-RNFL thickness, and whole superficial vascular density; nevertheless, there was 
TABLE 7 | Correlation between the MMSE, Fazekas scale, and OCTA parameters.

\begin{tabular}{lcccc}
\hline & $\begin{array}{c}\text { Deep whole } \\
\text { enface }\end{array}$ & Deep parafovea & $\begin{array}{c}\text { Superficial whole } \\
\text { enface }\end{array}$ & $\begin{array}{c}\text { Superficial } \\
\text { parafovea }\end{array}$ \\
\hline MMSE & & & & \\
$r$ & 0.03 & 0.057 & -0.072 & -0.066 \\
$p$-value & 0.744 & 0.537 & 0.441 & 0.474 \\
Fazekas scale & & & \\
$r$ & -0.073 & -0.149 & 0.04 & -0.007 \\
$p$-value & 0.644 & 0.374 & 0.804 & 0.962 \\
\hline
\end{tabular}

The correlation between cognition and OCTA parameters was evaluated using Pearson's correlation. MMSE, Mini-Mental State Examination; Fazekas scale, a 0-3 rating scale to quantify the amount of white matter T2 hyperintense lesions.

no significant correlation between global cognition (MMSE scores) or between the Fazekas score and retinal OCT angiogram flow density. Therefore, we hypothesized that the decrease in blood flow density might be the subsequent changes in blood flow caused by the atrophy of retinal ganglion cells and the reduction of oxygen consumption. Zabel et al. (2019b) studied the impairment of microvasculature in $\mathrm{AD}$ and primary open-angle glaucoma (POAG). They found that there were abnormalities throughout the entire retinal vascular system in both diseases; significant microcirculatory impairment in POAG patients affects superficial vessels. These findings further suggest that changes in retinal vasculature might be present in the prodromal stage of AD, namely, MCI (Zabel et al., 2019b). As the current studies have a cross-sectional design, the causation between disease and blood flow cannot be determined. Querques et al. (2019) demonstrated that $\mathrm{AD}$ and MCI patients are characterized by significant impairment of retinal neurovascular coupling, and this impairment is inversely related to the level of amyloid $\beta$ in the CSF, but they did not find differences in OCTA. Another reason might be the deposition of amyloid $\beta$ peptide $(A \beta)$ in the retinal blood vessel wall, which could impair the vasculature of both the brain and the retina.

Some researchers found that both clinical and preclinical AD patients showed an expanded FAZ area (O'Bryhim et al., 2018; Zabel et al., 2019b). However, some research showed that there was no change in the FAZ area (Lahme et al., 2018). No difference in the FAZ areas was observed among the three groups in our study. It is known that the FAZ varies significantly among individuals. Even in healthy individuals, FAZ parameters have high variability (Curcio, 2018; Linderman et al., 2018). Factors such as age, race, sex, and axial length can affect the size of the FAZ region (Wagner-Schuman et al., 2011; Sampson et al., 2017). Therefore, the FAZ may not be a sensitive measure. In the same way, it has been pointed out that direct comparison of individual FAZ areas is not very sensitive, and it may be more reliable to observe the individual change in the FAZ over time (Mammo et al., 2015). Considering the differences and changes in the FAZ in individuals, we will follow up with patients in a future study to obtain more robust data.

The current study has some limitations. First, although we established the diagnosis based on a standard clinical procedure, PET or CSF biomarkers were not included, and this could limit the precision level of our diagnosis. Additionally, because of the lack of detection of markers related to amyloid and tau proteins, we cannot analyze the correlation between them and OCT measurements. Second, although there was no significant difference in age among the three groups and the influence of age was corrected through multivariate analysis, the healthy control group was younger than the $\mathrm{MCI}$ and $\mathrm{AD}$ groups. In future studies, we still need to include older healthy subjects to control for the possible influence of age. In the meantime, both POAG open-angle glaucoma and MCI are chronic, age-related neurodegenerative changes. Glaucoma is one of the risk factors for AD (Chen et al., 2018; Lee et al., 2019; Xu et al., 2019). Zabel et al.'s (2019a) study found that the nerve fiber layer thickness in glaucoma patients without visual field change was similar to that in $\mathrm{AD}$ patients. Therefore, although we excluded individuals with high IOP and angle-closure glaucoma in our study, we cannot wholly exclude normal intraocular tension glaucoma, which also requires long-term follow-up. In addition, at present, there are no standardized protocols for OCTA image acquisition; the lack of defined protocols may produce inconsistencies in clinical practice. Some scholars have suggested that the quality of the image might be reduced if the same number of B-scans were used in a widened field of scanning (de Carlo et al., 2015). Therefore, B-scans covering an area of $3 \times 3 \mathrm{~mm}^{2}$, repeated horizontally and vertically, were used in our study to analyze the macula area. In a previous article (Haan et al., 2019), a correlation between vascular density and quality factor was observed in OCTA scans. A lower quality factor was associated with lower vascular density. However, in this study, we controlled for image quality by using only those with an OCTA image quality above 6. The effect of image quality on the results needs to be considered carefully. On the other hand, our data show that the differences in macular vascular density among controls, MCI and AD patients are not as distinct. Therefore, the use of vascular density currently might not be sufficiently reliable. Finally, due to the study design, we cannot eliminate the possibility of retinal changes over time.

In summary, the current study demonstrates that patients with $\mathrm{AD}$ and MCI have a reduced superficial retinal flow density under OCT angiography, suggesting that retinal microvascular dysfunction occurs in MCI and AD. Future studies will be focused on longitudinal follow-up with larger sample sizes.

\section{DATA AVAILABILITY STATEMENT}

The original contributions presented in the study are included in the article/supplementary material, further inquiries can be directed to the corresponding author/s.

\section{ETHICS STATEMENT}

The studies involving human participants were reviewed and approved by Huashan Hospital Institutional Review Board (HIRB), Fudan University (Shanghai). The patients/participants provided their written informed consent to participate in this study. 


\section{AUTHOR CONTRIBUTIONS}

$\mathrm{XW}, \mathrm{QZ}, \mathrm{ZL}$, and YX contributed to the conception and design of the study. XW, RT, and YM organized the database. XW and QZ performed the statistical analysis. HL, ZX, LZ, DD, and SD contributed to $\mathrm{AD}$ and $\mathrm{MCI}$ assessment and recruitment. All authors contributed to manuscript revision, read, and approved the submitted version.

\section{REFERENCES}

Albert, M. S., DeKosky, S. T., Dickson, D., Dubois, B., Feldman, H. H., Fox, N. C., et al. (2011). The diagnosis of mild cognitive impairment due to Alzheimer's disease: recommendations from the National Institute on AgingAlzheimer's Association workgroups on diagnostic guidelines for Alzheimer's disease. Alzheimers Dement. 7, 270-279. doi: 10.1016/j.jalz.2011.03.008

Arvanitakis, Z., Capuano, A. W., Leurgans, S. E., Bennett, D. A., and Schneider, J. A. (2016). Relation of cerebral vessel disease to Alzheimer's disease dementia and cognitive function in elderly people: a cross-sectional study. Lancet Neurol. 15, 934-943. doi: 10.1016/s1474-4422(16)30029-1

Berisha, F., Feke, G. T., Trempe, C. L., McMeel, J. W., and Schepens, C. L. (2007). Retinal abnormalities in early Alzheimer's disease. Invest. Ophthalmol. Vis. Sci. 48, 2285-2289. doi: 10.1167/iovs.06-1029

Bulut, M., Kurtulus, F., Gozkaya, O., Erol, M. K., Cengiz, A., Akidan, M., et al. (2018). Evaluation of optical coherence tomography angiographic findings in Alzheimer's type dementia. Br. J. Ophthalmol. 102, 233-237. doi: 10.1136/ bjophthalmol-2017-310476

Chen, Y. Y., Lai, Y. J., Yen, Y. F., Shen, Y. C., Wang, C. Y., Liang, C. Y., et al. (2018). Association between normal tension glaucoma and the risk of Alzheimer's disease: a nationwide population-based cohort study in Taiwan. BMJ Open. 8:e022987. doi: 10.1136/bmjopen-2018-022987

Cheung, C. Y., Ong, Y. T., Hilal, S., Ikram, M. K., Low, S., Ong, Y. L., et al. (2015). Retinal ganglion cell analysis using high-definition optical coherence tomography in patients with mild cognitive impairment and Alzheimer's disease. J. Alzheimers Dis. 45, 45-56. doi: 10.3233/jad-14 1659

Curcio, C. A. (2018). Viewing retinal vasculature in Alzheimer disease. JAMA Ophthalmol. 136, 1249-1250. doi: 10.1001/jamaophthalmol.2018.3569

de Carlo, T. E., Romano, A., Waheed, N. K., and Duker, J. S. (2015). A review of optical coherence tomography angiography (OCTA). Int. J. Retina Vitr. 1:5. doi: 10.1186/s40942-015-0005-8

den Haan, J., Balk, L. J., and Verbraak, F. D. (2018). Ganglion cell layer measurements correlate with disease severity in patients with Alzheimer's disease. Acta Ophthalmol. 96:e00265-66. doi: 10.1111/aos.13550

den Haan, J., Csinscik, L., Parker, T., Paterson, R. W., Slattery, C. F., Foulkes, A., et al. (2019a). Retinal thickness as potential biomarker in posterior cortical atrophy and typical Alzheimer's disease. Alzheimers Res. Ther. 11:62. doi: 10. 1186/s13195-019-0516-x

den Haan, J., van de Kreeke, J. A., Konijnenberg, E., ten Kate, M., den Braber, A., Barkhof, F., et al. (2019b). Retinal thickness as a potential biomarker in patients with amyloid-proven early- and late-onset Alzheimer's disease. Alzheimers Dement. 11, 463-471. doi: 10.1016/j.dadm.2019.05.002

Fazekas, F., Chawluk, J. B., Alavi, A., Hurtig, H. I., and Zimmerman, R. A. (1987). MR signal abnormalities at $1.5 \mathrm{~T}$ in Alzheimer's dementia and normal aging. Am. J. Neuroradiol. 8, 421-426.

Feke, G. T., Hyman, B. T., Stern, R. A., and Pasquale, L. R. (2015). Retinal blood flow in mild cognitive impairment and Alzheimer's disease. Alzheimers Dement. 1, 144-151. doi: 10.1016/j.dadm.2015.01.004

Haan, J. D., van de Kreeke, J. A., van Berckel, B. N., Barkhof, F., Teunissen, C. E., Scheltens, P., et al. (2019). Is retinal vasculature a biomarker in amyloid proven Alzheimer's disease? Alzheimers Dement. 11, 383-391. doi: 10.1016/j. dadm.2019.03.006

Handels, R. L. H., Wimo, A., Dodel, R., Kramberger, M. G., Visser, P. J., Molinuevo, J. L., et al. (2017). Cost-utility of using Alzheimer's disease biomarkers in cerebrospinal fluid to predict progression from mild cognitive

\section{FUNDING}

This work was supported by grants from National Chronic Disease Project (2016YFC1306402), Science and Technology Commission of Shanghai Municipality (19411961700, 17411950106, 2018SHZDZX03, and 17411950701), and National Natural Science Foundation of China (81773513).

impairment to dementia. J. Alzheimers Dis. 60, 1477-1487. doi: 10.3233/jad-1 70324

Hansson, O., Buchhave, P., Zetterberg, H., Blennow, K., Minthon, L., and Warkentin, S. (2009). Combined rCBF and CSF biomarkers predict progression from mild cognitive impairment to Alzheimer's disease. Neurobiol. Aging 30, 165-173. doi: 10.1016/j.neurobiolaging.2007.06.009

Hays, C. C., Zlatar, Z. Z., and Wierenga, C. E. (2016). The utility of cerebral blood flow as a biomarker of preclinical Alzheimer's disease. Cell Mol. Neurobiol. 36, 167-179. doi: 10.1007/s10571-015-0261-Z

Hirao, K., Ohnishi, T., Hirata, Y., Yamashita, F., Mori, T., Moriguchi, Y., et al. (2005). The prediction of rapid conversion to Alzheimer's disease in mild cognitive impairment using regional cerebral blood flow SPECT. Neuroimage 28, 1014-1021. doi: 10.1016/j.neuroimage.2005.06.066

Jia, L., Quan, M., Fu, Y., Zhao, T., Li, Y., Wei, C., et al. (2020). Dementia in China: epidemiology, clinical management, and research advances. Lancet Neurol. 19, 81-92. doi: 10.1016/s1474-4422(19)30290-x

Jiang, H., Wei, Y., Shi, Y., Wright, C. B., Sun, X., Gregori, G., et al. (2018). Altered macular microvasculature in mild cognitive impairment and Alzheimer disease. J. Neuroophthalmol. 38, 292-298. doi: 10.1097/wno.0000000000000580

Karakosta, A., Vassilaki, M., Plainis, S., Elfadl, N. H., Tsilimbaris, M., and Moschandreas, J. (2012). Choice of analytic approach for eye-specific outcomes: one eye or two?. Am. J. Ophthalmol. 153, 571-579.e571. doi: 10.1016/j.ajo.2011. 08.032

Kesler, A., Vakhapova, V., Korczyn, A. D., Naftaliev, E., and Neudorfer, M. (2011). Retinal thickness in patients with mild cognitive impairment and Alzheimer's disease. Clin. Neurol. Neurosurg. 113, 523-526. doi: 10.1016/j.clineuro.2011. 02.014

Lahme, L., Esser, E. L., Mihailovic, N., Schubert, F., Lauermann, J., Johnen, A., et al. (2018). Evaluation of ocular perfusion in alzheimer's disease using optical coherence tomography angiography. J. Alzheimers Dis. 66, 1745-1752. doi: $10.3233 /$ jad- 180738

Langa, K. M., and Levine, D. A. (2014). The diagnosis and management of mild cognitive impairment: a clinical review. JAMA 312, 2551-2561. doi: 10.1001/ jama.2014.13806

Lee, C. S., Larson, E. B., Gibbons, L. E., Lee, A. Y., McCurry, S. M., Bowen, J. D., et al. (2019). Associations between recent and established ophthalmic conditions and risk of Alzheimer's disease. Alzheimers Dement. 15, 34-41. doi: 10.1016/j.jalz.2018.06.2856

Linderman, R. E., Muthiah, M. N., Omoba, S. B., Litts, K., Tarima, S., Visotcky, A., et al. (2018). Variability of foveal avascular zone metrics derived from optical coherence tomography angiography images. Transl. Vis. Sci. Technol. 7:20. doi: $10.1167 /$ tvst.7.5.20

Livingston, G., Sommerlad, A., Orgeta, V., Costafreda, S. G., Huntley, J., Ames, D., et al. (2017). Dementia prevention, intervention, and care. Lancet 390, 2673-2734. doi: 10.1016/s0140-6736(17)31363-6

Mammo, Z., Balaratnasingam, C., Yu, P., Xu, J., Heisler, M., Mackenzie, P., et al. (2015). Quantitative noninvasive angiography of the fovea centralis using speckle variance optical coherence tomography. Invest. Ophthalmol. Vis. Sci. 56, 5074-5086. doi: 10.1167/iovs.15-16773

Marziani, E., Pomati, S., Ramolfo, P., Cigada, M., Giani, A., Mariani, C., et al. (2013). Evaluation of retinal nerve fiber layer and ganglion cell layer thickness in Alzheimer's disease using spectral-domain optical coherence tomography. Invest. Ophthalmol. Vis. Sci. 54, 5953-5958. doi: 10.1167/iovs.13-12046

Morris, J. C., Storandt, M., Miller, J. P., McKeel, D. W., Price, J. L., Rubin, E. H., et al. (2001). Mild cognitive impairment represents early-stage Alzheimer disease. Arch Neurol. 58, 397-405. 
O'Bryhim, B. E., Apte, R. S., Kung, N., Coble, D., and Van Stavern, G. P. (2018). Association of preclinical Alzheimer disease with optical coherence tomographic angiography findings. JAMA Ophthalmol. 136, 1242-1248. doi: 10.1001/jamaophthalmol.2018.3556

Ottoy, J., Niemantsverdriet, E., Verhaeghe, J., De Roeck, E., Struyfs, H., Somers, C., et al. (2019). Association of short-term cognitive decline and MCI-to-AD dementia conversion with CSF, MRI, amyloid- and (18)F-FDG-PET imaging. Neuroimage Clin. 22:101771. doi: 10.1016/j.nicl.2019.101771

Park, K. W., Yoon, H. J., Kang, D. Y., Kim, B. C., Kim, S., and Kim, J. W. (2012). Regional cerebral blood flow differences in patients with mild cognitive impairment between those who did and did not develop Alzheimer's disease. Psychiatry Res. 203, 201-206. doi: 10.1016/j.pscychresns.2011.12.007

Pillai, J. A., Bermel, R., Bonner-Jackson, A., Rae-Grant, A., Fernandez, H., Bena, J., et al. (2016). Retinal nerve fiber layer thinning in Alzheimer's disease: a case-control study in comparison to normal aging, parkinson's disease, and non-alzheimer's dementia. Am. J. Alzheimers Dis. Other Dement. 31, 430-436. doi: $10.1177 / 1533317515628053$

Querques, G., Borrelli, E., Sacconi, R., De Vitis, L., Leocani, L., Santangelo, R., et al. (2019). Functional and morphological changes of the retinal vessels in Alzheimer's disease and mild cognitive impairment. Sci. Rep. 9:63. doi: 10.1038/ s41598-018-37271-6

Rensma, S. P., van Sloten, T. T., Launer, L. J., and Stehouwer, C. D. A. (2018). Cerebral small vessel disease and risk of incident stroke, dementia and depression, and all-cause mortality: a systematic review and meta-analysis. Neurosci. Biobehav. Rev. 90, 164-173. doi: 10.1016/j.neubiorev.2018.04.003

Sampson, D. M., Gong, P., An, D., Menghini, M., Hansen, A., Mackey, D. A., et al. (2017). Axial length variation impacts on superficial retinal vessel density and foveal avascular zone area measurements using optical coherence tomography angiography. Invest. Ophthalmol. Vis. Sci. 58, 3065-3072. doi: 10.1167/iovs.1721551

Sánchez, D., Castilla-Marti, M., Marquié, M., Valero, S., Moreno-Grau, S., Rodríguez-Gómez, O., et al. (2020). Evaluation of macular thickness and volume tested by optical coherence tomography as biomarkers for Alzheimer's disease in a memory clinic. Sci. Rep. 10:1580. doi: 10.1038/s41598-020-58399-4

Scheltens, P., Erkinjunti, T., Leys, D., Wahlund, L. O., Inzitari, D., del Ser, T., et al. (1998). White matter changes on CT and MRI: an overview of visual rating scales. European Task force on age-related white matter changes. Eur. Neurol. 39, 80-89. doi: 10.1159/000007921

Smith, E. E., and Beaudin, A. E. (2018). New insights into cerebral small vessel disease and vascular cognitive impairment from MRI. Curr. Opin. Neurol. 31, 36-43. doi: 10.1097/wco.0000000000000513

Sperling, R. A., Aisen, P. S., Beckett, L. A., Bennett, D. A., Craft, S., Fagan, A. M., et al. (2011). Toward defining the preclinical stages of Alzheimer's disease: recommendations from the National Institute on Aging-Alzheimer's Association workgroups on diagnostic guidelines for Alzheimer's disease. Alzheimers Dement. 7, 280-292. doi: 10.1016/j.jalz.2011.03.003

Tao, R., Lu, Z., Ding, D., Fu, S., Hong, Z., Liang, X., et al. (2019). Perifovea retinal thickness as an ophthalmic biomarker for mild cognitive impairment and early Alzheimer's disease. Alzheimers Dement. 11, 405-414. doi: 10.1016/j. dadm.2019.04.003

Trebbastoni, A., D'Antonio, F., Bruscolini, A., Marcelli, M., Cecere, M., Campanelli, A., et al. (2016). Retinal nerve fibre layer thickness changes in
Alzheimer's disease: results from a 12-month prospective case series. Neurosci. Lett. 629, 165-170. doi: 10.1016/j.neulet.2016.07.006

van de Kreeke, J. A., Nguyen, H. T., Konijnenberg, E., Tomassen, J., den Braber, A., Ten Kate, M., et al. (2020). Optical coherence tomography angiography in preclinical Alzheimer's disease. Br. J. Ophthalmol. 104, 157-161. doi: 10.1136/ bjophthalmol-2019-314127

van der Flier, W. M., Skoog, I., Schneider, J. A., Pantoni, L., Mok, V., Chen, C. L. H., et al. (2018). Vascular cognitive impairment. Nat. Rev. Dis. Primers 4:18003. doi: $10.1038 /$ nrdp. 2018.3

Vos, S. J., Verhey, F., Frolich, L., Kornhuber, J., Wiltfang, J., Maier, W., et al. (2015). Prevalence and prognosis of Alzheimer's disease at the mild cognitive impairment stage. Brain 138(Pt 5), 1327-1338. doi: 10.1093/brain/awv029

Wagner-Schuman, M., Dubis, A. M., Nordgren, R. N., Lei, Y., Odell, D., Chiao, H., et al. (2011). Race- and sex-related differences in retinal thickness and foveal pit morphology. Invest. Ophthalmol. Vis. Sci. 52, 625-634. doi: 10.1167/iovs.105886

Xu, X. H., Zou, J. Y., Geng, W., and Wang, A. Y. (2019). Association between glaucoma and the risk of Alzheimer's disease: a systematic review of observational studies. Acta Ophthalmol. 97, 665-671. doi: 10.1111/aos. 14114

Xu, Y., Chen, K., Zhao, Q., Li, F., and Guo, Q. (2018). Short-term delayed recall of auditory verbal learning test provides equivalent value to long-term delayed recall in predicting MCI clinical outcomes: a longitudinal follow-up study. Appl. Neuropsychol. Adult. 27, 73-81. doi: 10.1080/23279095.2018.148 1067

Yamashita, K. I., Taniwaki, Y., Utsunomiya, H., and Taniwaki, T. (2014). Cerebral blood flow reduction associated with orientation for time in amnesic mild cognitive impairment and Alzheimer disease patients. J. Neuroimaging 24, 590-594. doi: 10.1111/jon.12096

Zabel, P., Kaluzny, J. J., Wilkosc-Debczynska, M., Gebska-Toloczko, M., Suwala, K., Kucharski, R., et al. (2019a). Peripapillary retinal nerve fiber layer thickness in patients with Alzheimer's disease: a comparison of eyes of patients with Alzheimer's disease, primary open-angle glaucoma, and preperimetric glaucoma and healthy controls. Med. Sci. Monit. 25, 1001-1008. doi: 10.12659/ msm.914889

Zabel, P., Kaluzny, J. J., Wilkosc-Debczynska, M., Gebska-Toloczko, M., Suwala, K., Zabel, K., et al. (2019b). Comparison of retinal microvasculature in patients with Alzheimer's disease and primary open-angle glaucoma by optical coherence tomography angiography. Invest. Ophthalmol. Vis. Sci. 60, 34473455. doi: 10.1167/iovs.19-27028

Conflict of Interest: The authors declare that the research was conducted in the absence of any commercial or financial relationships that could be construed as a potential conflict of interest.

Copyright (c) 2021 Wang, Zhao, Tao, Lu, Xiao, Zheng, Ding, Ding, Ma, Lu and Xiao. This is an open-access article distributed under the terms of the Creative Commons Attribution License (CC BY). The use, distribution or reproduction in other forums is permitted, provided the original author(s) and the copyright owner(s) are credited and that the original publication in this journal is cited, in accordance with accepted academic practice. No use, distribution or reproduction is permitted which does not comply with these terms. 